\title{
Assessment of heavy metals in water, sediment and fish tissues, from Sharkia province, Egypt
}

\section{El-Sayed A. El-Sayed ${ }^{1}$, Mohamed S. El-Ayyat ${ }^{2}$, El-Sayed Nasr ${ }^{1}$ and Zeinab Z. K. Khater ${ }^{1}$}

1- Department of Zoology, Faculty of Science, Zagazig University, Egypt

2- Department of Animal Production, Faculty of Agriculture, Zagazig University, Egypt

\begin{abstract}
$\mathrm{T}$ he present study was conducted to investigate factors affecting live fish at different sources of water (agriculture drainage, industrial drainage and sewage wastewater). Heavy metal residues (zinc, copper, lead, cadmium, chromium, aluminium, manganese, nikel and cobalt) were determined in water, sediment and fish organs (muscles, gills, kidney and liver tissues) of three fishes (Oreochromis niloticus, Clarias gariepinus and Bagrus bayad). The industrial drainage and sewage water revealed the highest concentrations of heavy metals followed by agriculture drainage water. The muscles, gills, kidney and liver tissues of fish samples collected from industrial drainage and sewage canals had the highest levels of heavy metal residues followed by agriculture drainage canals. The heavy metal studies revealed that there is a public health hazard associated with industrial drainage, sewage wastewater and agriculture drainage as the quality of fish did not comply with the standard levels recommended by WHO, USEPA and ANZECC. Potential adverse health effects in such applications could be avoided if the wastewater is sufficiently treated.
\end{abstract}

Keywords: water, sediment, fish tissue, heavy metals, Tilapia, Catfish.

\section{INTRODUCTION}

The pollution of aquatic ecosystems by heavy metals is an important environmental problem, as heavy metals constitute some of the most hazardous substances that can bioaccumulate in various biotic systems. Bioaccumulation is a process in which a chemical pollutant enters into the body of an organism and is not excreted, but accumulated in the organism's tissues. Metals that are deposited in the aquatic environment may accumulate in the food chain and cause ecological damage, while also posing a threat to human health. Cancer and damage of the nervous system have been documented in humans as a result of metal consumption. The U. S. Environmental Protection Agency conducted a national study of accumulated toxins documenting this concern (Van den Broek et al., 2002).

Anthropogenic impacts including industrial discharge, domestic sewage, non-point source runoff and atmospheric precipitation are the main sources of 
toxic heavy metals that enter aquatic systems (Langston et al., 1999).However, metals also occur in small amounts naturally and enter aquatic systems through ore-bearing rocks, wind-blow dust, forest fires and vegetation (FernandezLeborans and Olalla-Herrero, 2000).

The heavy metal pollution of aquatic ecosystems is often most obvious in sediments , macrophytes and aquatic animals, than in elevated concentrations in water (Linnik and Zubenko, 2000). Therefore aquatic ecosystems are typically monitored for pollution of heavy metals using biological assays (Wong and Dixon, 1995).Many aquatic organisms have been used as bioindicators, including aquatic insects (Rayms-Keller et al., 1998), plants (Mohan and Hosetti, 1999), protozoans (Fernandez-Leborans and Olalla-Herrero, 2000), crustaceans (Allinson et al., 2000) and fish (Burger et al., 2002).

Fish species are often the top consumers in aquatic ecosystems (Dallinger et al., 1987) and thus metal concentrations in fish can act as an environmental indicator for the environmental state( Wildianarko et al., 2000). Fish are known to bioaccumulate metals and therefore can be used as biomonitors, also they have the advandage of allowing the comparison of metal concentrations among sites, where water samples are near or below the detection limits of the atomic absorption technique (Ramelow et al., 1989).

The mechanisms of metal toxicity to fish are varied, although many act as enzyme poisons. Therefore, it is difficult to assess the probable effect of a measured concentration of a metal. In pond water heavy metals can be adsorbed onto clay particles and chelated by organic matter so that they remain in solution but may not have an adverse effect on fish or crustaceans (Boyd, 1990).

\section{1- Description of the investigated areas:}

Fish samples were collected from five localities at Sharkia Province that are considered as natural sources for fishery. These localities lie east to Damietta Branch (Nile water). The five localities are: Kafr El-Hosr pond (at Kafr El-Hosr Bridge), Muweis canal (Zagazig canal at ADab Bridge near oil and soap factory), Bilbeis canal (at Bilbeis boat), Abbassa canal (Ismaillya canal at Ismaillya boat) and San El-Hagar canal (Faqus canal at south San El-Hagar Bridge). Kafr El-Hosr pond lies at the north of Zagazig city and was selected as a polluted area; where it receives several sources of pollution, as domestic and agricultural wastes. Muweis canal lies at the centre of Zagazig city and was selected as a polluted area; where it receives industrial wastes from oil and soap factory. Bilbeis canal lies at east of Muweis canal and was selected as a polluted area; where it receives agricultural wastes. Abbassa canal lies also at the east of Muweis canal and was selected as a polluted area (agricultural drainage area). San El- Hagar canal lies at the north of Faqus city and was selected as a polluted area; where it receives domestic and agricultural wastes. 


\section{2- Sampling sites:}

Samples were collected monthly during the period from September 2007 to August 2008 at Kafr El-Hosr pond, Muweis canal, Bilbeis canal, Abbassa canal and San El-Hagar canal. A large number of peoples are visiting the above sites for heavy fishing, so these areas are suffering severe disturbance from human activity (agricultural, industrial, domestic or fishing).

\section{3- The collected fish:}

Oreochromis niloticus is the most economic fish in Egypt. Its production in 2003 was about 200000 tons. It is greenish-olive, shining silvery in color. Dorsal, anal and caudal fins may be edged with bright red. A dark spot appears on the operculum (Rakocy, 2011).

Clarias gariepinus is one of the most important freshwater fishes in Egypt. Total production of it in 2007 was about 31.9 thousand tons; i.e. it contributes about $17.5 \%$ of the total Nile catch in Egypt. It is grayish olive to olive brown to blackish above, white or grayish beneath (Abdel-Hafez and El-Caryony, 2009).

Bagrus bayad is well marketable. Its total production in 1996 of about 5826 tons; i.e. it contributes about $9 \%$ of the total Nile catch. It is silvery grey above, white beneath and its fins are colorless. Dark dots are sometimes scattered on the back, the adipose and the caudal fins (Bishai and Khalil, 1997).

\section{4- Analytical procedures:}

Water, sediments and fish samples (muscles, gills, kidney and liver of each fish) were taken monthly from each site and analyzed for heavy metal residues; copper $(\mathrm{Cu})$, zinc $(\mathrm{Zn})$, lead $(\mathrm{Pb})$, cadmium $(\mathrm{Cd})$, chromium $(\mathrm{Cr})$, manganese $(\mathrm{Mn})$, aluminium $(\mathrm{Al})$, nikel $(\mathrm{Ni})$ and cobalt $(\mathrm{Co})$. Water samples were collected and kept in $500 \mathrm{ml}$ sterile plastic containers while sediments and fish samples were collected and kept in plastic bags. Water samples for metal analysis was treated with $1 \mathrm{ml}$ of HCL in $500 \mathrm{ml}$ sample to arrest microbial activities.

\section{A-Water analysis:}

\section{* Heavy metals in water:}

Heavy metal concentrations in water were determined by atomic absorption spectrophotometer (Perkin Elmer, 2280). The samples were prepared and analyzed sequentialy for zinc, copper, lead, cadmium, chromium, manganese, aluminium, nikel and cobalt according to APHA (1985). To $50 \mathrm{ml}$ of unfiltered water sample (in $500 \mathrm{ml}$ Taylor flask) $0.50 \mathrm{ml}$ of concentrated sulphuric acid was added. This was boiled down to obtain white fumes, cooled and $1.0 \mathrm{ml}$ of $60 \% \mathrm{HCLO}_{3}$ and $5.0 \mathrm{ml}$ of concentrated $\mathrm{HNO}_{3}$ were added. The resulting mixture was digested until a clear digest was obtained. This was cooled, filtered (No. 44 Whatman paper) into $500 \mathrm{ml}$ volumetric flask, diluted to volume and mixed.

\section{Heavy metal concentration $(\mathbf{p p m})=$} reading of atomic absorption $\mathrm{X}$ volume of diluted solution 


\section{B- Sediment analysis:}

\section{* Heavy metals in sediments:}

Soil sample solution for metal analysis was prepared by treating $1 \mathrm{~g}$ soil sample with $10 \mathrm{ml}$ of concentrated nitric acid and $5 \mathrm{ml}$ of $60 \%$ perchloric acid in $100 \mathrm{ml} \mathrm{Kjeldahl} \mathrm{flask.} \mathrm{The} \mathrm{mixture} \mathrm{was} \mathrm{heated} \mathrm{with} \mathrm{moderate} \mathrm{heat} \mathrm{using} \mathrm{a} \mathrm{hot}$ plate for about 15 min until white fumes appear. The digest was cooled, then filtered (No. 44 whatman paper) into $50 \mathrm{ml}$ volumetric flask with rinsing in deionized water and made up to mark with de-ionized water (Akubugwo et al., 2007).

\section{Heavy metal concentration $(\mu \mathrm{g} / \mathrm{g})=$}

reading of atomic absorption $\mathrm{X}$ volume of diluted solution

\section{C-Fish analysis:}

Weight of sample (g.)

\section{* Heavy metals in fish tissues:}

Thirty fish from each species (Oreochromis niloticus, Clarias gariepinus and Bagrus bayad) were collected monthly from each site for heavy metal analysis. The collected fish were washed with deionized water, put in cleaned plastic bags and stored frozen until analysis was carried out.

One gram of the prepared tissue sample (wet weight) was subjected to digestion by adding $10 \mathrm{ml}$ of freshly prepared $1: 1$ concentrated $\mathrm{HNO}_{3}-\mathrm{HCLO}_{3}$ in beaker, covered with a watch glass till initial reaction subsided in about 1 hour and gently heated at $160{ }^{\circ} \mathrm{C}$ in a sand bath on a hot plate till reduction of volume to 2-5 $\mathrm{ml}$. The digests were allowed to cool and transferred to $25 \mathrm{ml}$ volumetric flasks and made up to mark with de-ionized water. The digests were kept in plastic bottles and latter heavy metal concentrations were determined using an atomic absorption spectrophotometer (Olaifa et al., 2004).

\section{D- Statistical analysis:}

The statistical analysis was performed using the analysis of variance (ANOVA) to determine the differences between treatments mean at significant level of 0.05. Standard errors were also estimated. All statistics were run on the computer using SPSS program. All graphics and tables were made by using Origin 7.5 and Microsoft word (2003). The methods for analysis of the results were done according to Bishop (1980) and McCreadie et al. (2006).

\section{RESULTS}

\section{A-Water analysis:}

\section{* Heavy metals in water:}

Comparing the average concentrations of heavy metals in the different study sites, the data recorded in Table (1) and Fig. (1) showed annual variations in heavy metal concentrations in water samples. The concentrations had the order: $\mathrm{Zn}>\mathrm{Al}>\mathrm{Cr}>\mathrm{Pb}>\mathrm{MN}>\mathrm{Co}>\mathrm{Cu}>\mathrm{Cd}>\mathrm{Ni}$. 
Table 1: Heavy metals concentration (mean $\pm \mathrm{SE}$ ) of water samples collected from the investigated sites from autumn 2007 to summer 2008.

\begin{tabular}{|c|c|c|c|c|c|}
\hline Sules & $\begin{array}{l}\text { Kafr El-Hosr } \\
\text { pond }\end{array}$ & Billbeis canal & Abbassa canal & $\begin{array}{l}\text { San El-Hagar } \\
\text { canal }\end{array}$ & Mureis canal \\
\hline Zinc ( $(\mathrm{kn})$ & $9.378 \pm 0.985$ & $7.662 \pm 0.387$ & $8.473 \pm 1.247$ & $8.822 \pm 1.240$ & $10.194 \pm 2.040$ \\
\hline Copper (Cu) & $0.920 \pm 0.134$ & $1.335 \pm 0.216$ & $1.327 \pm 0.490$ & $2.818 \pm 1.498$ & $1.981 \pm 0.859$ \\
\hline Cadmim(Cd) $\mu g / g$ & $0.864 \pm 0.096$ & $1.030 \pm 0.073$ & $1.060 \pm 0.084$ & $1.077 \pm 0.229$ & $1.031 \pm 0.067$ \\
\hline Chromium(Cr) $\mu g / g$ & $5.517 \pm 4.555$ & $10.246 \pm 9.026$ & $3.694 \pm 2.851$ & $3.440 \pm 2.309$ & $2.378 \pm 1.610$ \\
\hline Ahminnum(AI) $\mu g / g$ & $64.478 \pm 25.783$ & $55.568 \pm 13.530$ & $59.661 \pm 14.106$ & $46.702 \pm 9.778$ & $51.468 \pm 10.483$ \\
\hline Lead (PD) & $5.014 \pm 2.005$ & $4.398 \pm 1.399$ & $4.945 \pm 2.121$ & $4.554 \pm 1.002$ & $4.148 \pm 1.251$ \\
\hline Manganes (Mn) $\mu g / g$ & $0.804 \pm 0.074$ & $0.865 \pm 0.156$ & $0.847 \pm 0.125$ & $0.647 \pm 0.113$ & $0.658 \pm 0.087$ \\
\hline NAfl(t) & $0.398 \pm 0.086$ & $0.302 \pm 0.066$ & $0.301 \pm 0.062$ & $0.354 \pm 0.058$ & $0.328 \pm 0.066$ \\
\hline Cobalt(Co) & $6.296 \pm 3.316$ & $4.949 \pm 2.098$ & $5.757 \pm 2.896$ & $6.353 \pm 3.488$ & $6.275 \pm 3.425$ \\
\hline
\end{tabular}

**Means in the same row are not significantly different ( $p>0.05)$, using ANOVA.

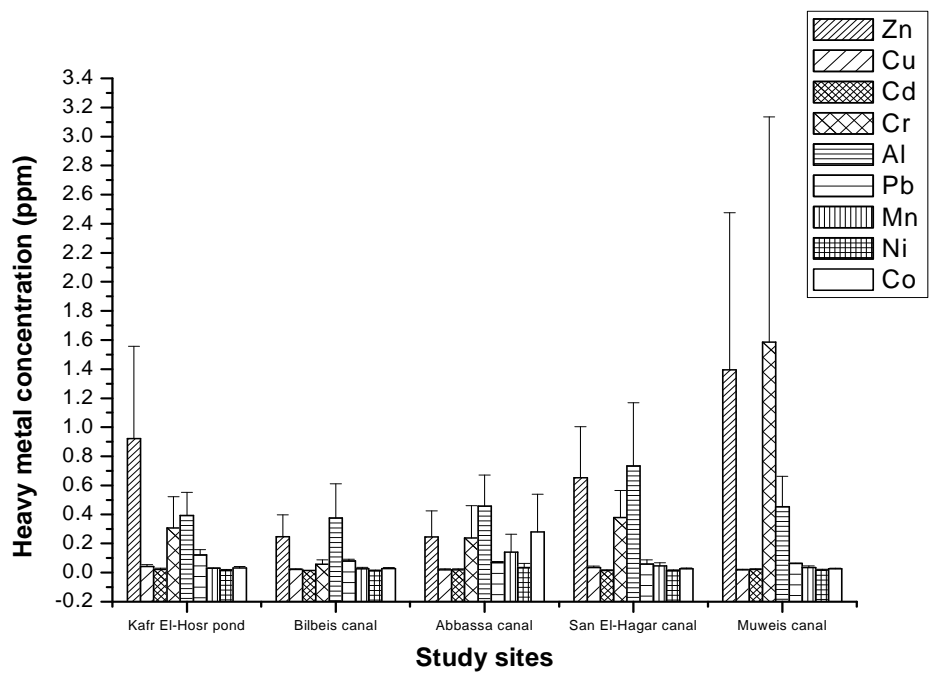

Fig. 1: Heavy metals concentrations (annual mean $\pm \mathrm{SE}$ ) in water samples collected from the different investigated sites at Sharkia province.

\section{B- Sediment analysis:}

\section{* Heavy metals in sediment:}

Comparing the average concentrations of heavy metals in the different study sites, the data recorded in Table (2) and Fig. (2) showed annual variations in heavy metal concentrations in sediment samples. The concentrations had the order: $\mathrm{Mn}>\mathrm{Zn}>\mathrm{Al}>\mathrm{Cr}>\mathrm{Cu}>\mathrm{Pb}>\mathrm{Co}>\mathrm{Ni}>\mathrm{Cd}$. 
Table 2: Heavy metals concentration (mean $\pm \mathrm{SE}$ ) of sediment samples collected from the investigated sites from autumn 2007 to summer 2008.

\begin{tabular}{|c|c|c|c|c|c|c|}
\hline Heavy metals & Silles & $\begin{array}{c}\text { Kafr El-Hosr } \\
\text { pond }\end{array}$ & Bitheis canal & Abbassa canal & $\begin{array}{c}\text { San El-Hagar } \\
\text { canal }\end{array}$ & Mureis canal \\
\hline Zlinc (Zn) & $\mu g / g$ & $14.894 \pm 1.320$ & $12.015 \pm 2.081$ & $11.538 \pm 2.917$ & $13.161 \pm 2.647$ & $16.893 \pm 1.587$ \\
\hline Copper (Cu) & $\mu g / g$ & $7.223 \pm 0.940$ & $5.339 \pm 1.310$ & $3.962 \pm 1.260$ & $6.156 \pm 1.862$ & 15 \\
\hline Lead (Pक) & $\mu \mathrm{g} g$ & $4.704 \pm 1.872$ & 2.48 & 2.3 & $3.764 \pm 1.298$ & 662 \\
\hline Cadminm (Cd) & $\mu \sigma / \mathrm{g}$ & $0.222 \pm 0.070$ & 0.14 & 0.18 & \pm 0.060 & 112 \\
\hline Chromium (C) & $\mu \sigma / \varepsilon$ & $11.725 \pm 5.603$ & 11.02 & .539 & $12.296 \pm 6.673$ & 6.568 \\
\hline Ahuminium (AI) & $\mu z / \xi$ & $15.196 \pm 7.052$ & $8.119 \pm 3.295$ & 5.75 & $19.309 \pm 7.956$ & \pm 4.264 \\
\hline Manganese( $(\mathrm{hn})$ & $\mu \not / \xi$ & $41.583 \pm 12.437$ & $51.680 \pm 18.696$ & $32.239 \pm 15.594$ & $37.459 \pm 16.972$ & $57.184 \pm 17.445$ \\
\hline NHal(K) & $\mu \mathrm{g} / \mathrm{g}$ & $2.035 \pm 0.139$ & $1.383 \pm 0.339$ & $1.144 \pm 0.500$ & $1.954 \pm 0.628$ & $2.330 \pm 0.260$ \\
\hline Cobalt(Co) & $\mu g / \mathrm{g}$ & $3.545 \pm 0.419$ & $3.223 \pm 1.309$ & $1.785 \pm 0.470 a$ & $2.821 \pm 0.647$ & $4.891 \pm 1.492 a$ \\
\hline
\end{tabular}

*Means with the same letters in the same row are significantly different $(\mathrm{p}<0.05)$, using ANOVA.

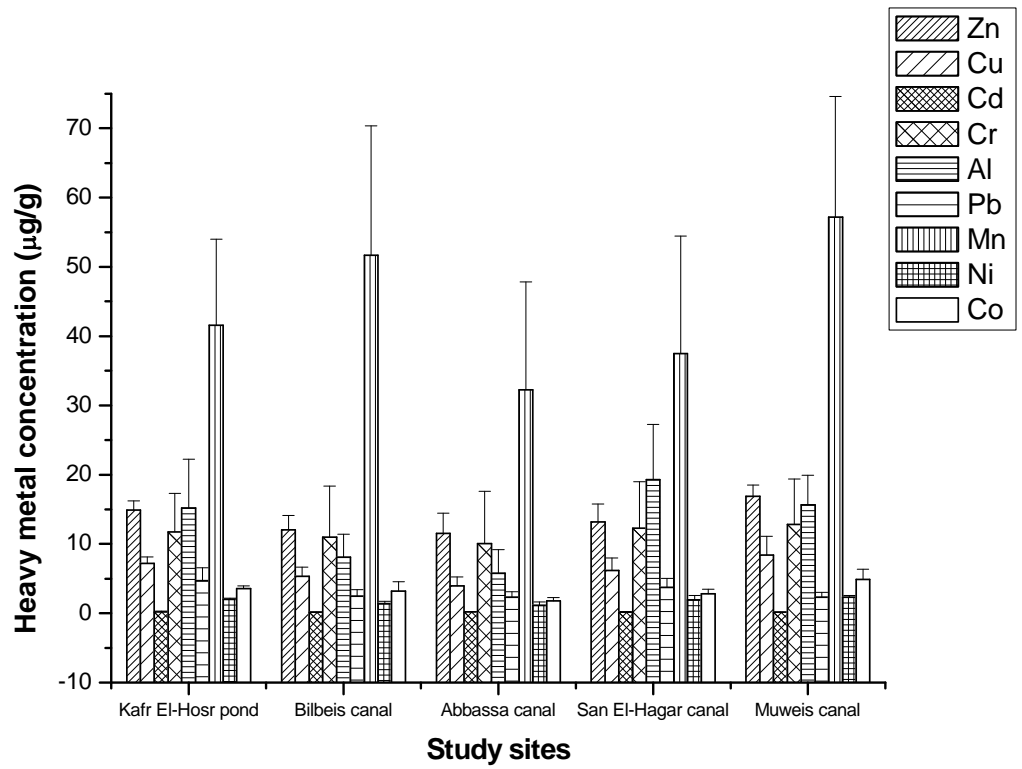

Fig. 2: Heavy metals concentration (annual mean $\pm \mathrm{SE}$ ) in sediment samples collected from the different investigated sites at Sharkia province.

\section{C- Fish analysis:}

\section{I- Heavy metals in fish muscle:}

Comparing the average concentrations of heavy metals in the different study sites, (Tables 3, 4 and 5) showed variations between heavy metal concentrations in fish muscle. The concentrations had the order: $\mathrm{Al}>\mathrm{Zn}>\mathrm{Co}>$ $\mathrm{Pb}>\mathrm{Cr}>\mathrm{Cd}>\mathrm{Mn}>\mathrm{Cu}>\mathrm{Ni}$. 
Table 3: Heavy metals concentration (mean $\pm \mathrm{SE}$ ) in muscle tissues of Oreochromis niloticus collected from the investigated sites from autumn 2007 to summer 2008.

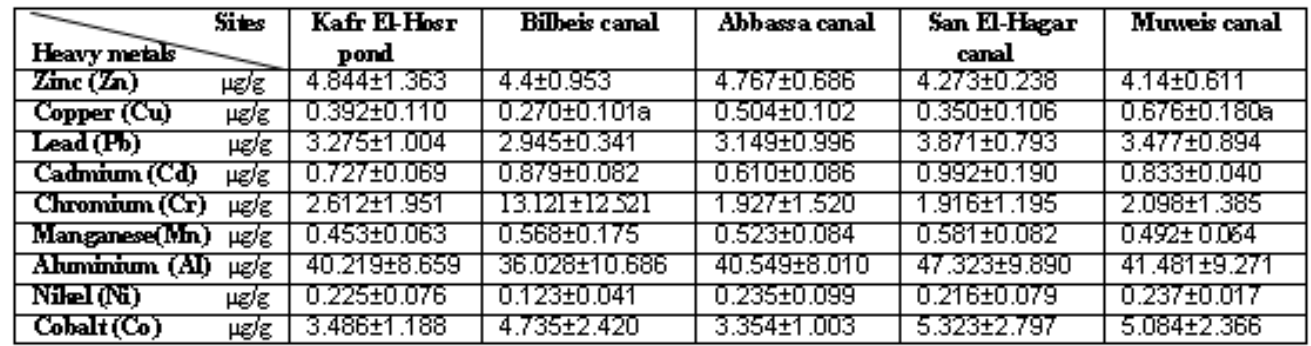

*Means with the same letters in the same row are significantly different $(\mathrm{p}<0.05)$, using ANOVA.

Table 4: Heavy metals concentration (mean $\pm \mathrm{SE}$ ) in muscle tissues of Clarias gariepinus collected from the investigated sites from autumn 2007 to summer 2008.

\begin{tabular}{|c|c|c|c|c|c|}
\hline Sintes & $\begin{array}{l}\text { Kafr El-Hoss } \\
\text { pond }\end{array}$ & Billbeis canal & Abbassa canal & $\begin{array}{c}\text { San El-Hagar } \\
\text { canal }\end{array}$ & Murueis canal \\
\hline Zhinc (Zn) & $5.310 \pm 1.189$ & $3.842 \pm 0.338$ & $4.405 \pm 0.603$ & $3.843 \pm 0.418$ & $5.674 \pm 0.819$ \\
\hline Copper (Cu) & $0.424 \pm 0.069$ & $0.159 \pm 0.038 \mathrm{a}$ & $0.545 \pm 0.208$ & $0.556 \pm 0.126$ & $0.899 \pm 0.3359$ \\
\hline Lead(P) & $4.200 \pm 1.685$ & $1.895 \pm 0.619$ & $3.349 \pm 1.281$ & $3.696 \pm 0.629$ & $3.443 \pm 1.014$ \\
\hline Cadminur(Cd) & $0.766 \pm 0.085$ & $0.648 \pm 0.201$ & $0.947 \pm 0.051$ & $0.946 \pm 0.215$ & $0.891 \pm 0.030$ \\
\hline Chromium (C) $\mu / \bar{c}$ & $3.737 \pm 3.240$ & $6684 \pm 5957$ & $1.798 \pm 1.308$ & $1.897 \pm 1.565$ & $1.412 \pm 1.086$ \\
\hline Hanganese(hin) $\mu / \mathrm{g}$ & $0.666 \pm 0.099$ & $0.465 \pm 0.130$ & $0.640 \pm 0.119$ & $0.439 \pm 0.068$ & $0522 \pm 0068$ \\
\hline Ahuminium(AI) $\mu g / g$ & $58.153 \pm 23.066$ & $39.288 \pm 9.504$ & $46.384 \pm 10.873$ & $41.980 \pm 8.806$ & $46.461 \pm 8.908$ \\
\hline Nife(Ni) & $0.303 \pm 0.075$ & $0.187 \pm 0.085$ & $0.155 \pm 0.050$ & $0.188 \pm 0.070$ & $0.239 \pm 0.072$ \\
\hline Cobalt(Co) & $5.666 \pm 3.074$ & $3.688 \pm 2.194$ & $4.827 \pm 2.348$ & $4.784 \pm 2.440$ & $5.660 \pm 3.046$ \\
\hline
\end{tabular}

*Means with the same letters in the same row are significantly different $(\mathrm{p}<0.05)$, using ANOVA.

Table 5: Heavy metals concentration (mean $\pm \mathrm{SE}$ ) in muscle tissues of Bagrus bayad collected from the investigated sites from autumn 2007 to summer 2008.

\begin{tabular}{|c|c|c|c|c|c|}
\hline Heavy metals & $\begin{array}{l}\text { Kafr E1-Hosr } \\
\text { pond }\end{array}$ & Bilbeis canal & Abbassa canal & $\begin{array}{c}\text { San El-Hagar } \\
\text { canal }\end{array}$ & Huweis canal \\
\hline Zfme $(\mathrm{Zn})$ & $4.718 \pm 1.863$ & $3.674 \pm 0.231$ & $3.766 \pm 0.285$ & $3.139 \pm 0.985$ & $3.033 \pm 1.076$ \\
\hline Lead(Pb) & $2.485 \pm 0.795$ & $3.010 \pm 0.720$ & $2.920 \pm 0.806$ & $1.845 \pm 0.238$ & $3.858 \pm 1.951$ \\
\hline Cadmím [Cd] & $0.802 \pm 0.006$ & $0.723 \pm 0.075$ & $0.726 \pm 0.089$ & $0.811 \pm 0.193$ & $0.893 \pm 0.128$ \\
\hline Chromium (Cr) $\mu g / g$ & $0.385 \pm 0.104$ & $6.493 \pm 6031$ & $2.018 \pm 1.485$ & $0.679 \pm 0.424$ & $1.610 \pm 0.988$ \\
\hline Ahmminum(A) $\mu g / g$ & $36.745 \pm 18.705$ & $28.578 \pm 10.145$ & $41.303 \pm 14.651$ & $43.842 \pm 9.735$ & $47.518 \pm 15.955$ \\
\hline NHal(1) & $0.164 \pm 0.146$ & $0.230 \pm 0.027 a$ & $0.085 \pm 0.016 a b$ & $0.239 \pm 0.0306$ & $0.206 \pm 0.062$ \\
\hline Cobalt (Co) & $7.965 \pm 5.415$ & $4.401 \pm 1.842$ & $4.857 \pm 2.555$ & $6.320 \pm 2.564$ & $5.080 \pm 2.659$ \\
\hline
\end{tabular}

*Means with the same letters in the same row are significantly different $(p<0.05)$, using ANOVA.

\section{II- Heavy metals in gill tissue:}

Comparing the average concentrations of heavy metals in the different study sites, (Tables 6, 7 and 8) showed variations between heavy metal concentrations in gill tissue. The concentration was as seen $\mathrm{Al}>\mathrm{Zn}>\mathrm{Co}>\mathrm{Pb}>$ $\mathrm{Cr}>\mathrm{Cd}>\mathrm{Mn}>\mathrm{Cu}>\mathrm{Ni}$. 
Table 6: Heavy metals concentration (mean $\pm \mathrm{SE}$ ) in gills of Oreochromis niloticus collected from the investigated sites from autumn 2007 to summer 2008.

\begin{tabular}{|c|c|c|c|c|c|c|}
\hline$\underbrace{\text { Sintes }}_{\text {etals }}$ & & $\begin{array}{l}\text { Kafr El-Hosr } \\
\text { pond }\end{array}$ & Billbeis canal & $\begin{array}{l}\text { Abbassa } \\
\text { canal }\end{array}$ & $\begin{array}{l}\text { San El-Hagar } \\
\text { canal }\end{array}$ & Murueis canal \\
\hline Znc (Zn) & $\mu g / \mathrm{g}$ & $9.293 \pm 4.505$ & $6.499 \pm 1.038$ & $5.901 \pm 0.749$ & $6.035 \pm 0.690$ & $6.800 \pm 0.610$ \\
\hline Copper (Cu) & $\mu \varepsilon / \xi$ & $0.572 \pm 0.122$ & $0.664 \pm 0.286$ & $0.623 \pm 0.076$ & $1.720 \pm 1.224$ & $1.063 \pm 0.315$ \\
\hline Cadmium (Cd) & $\mu 8 / \mathrm{g}$ & $0.858 \pm 0.100$ & $0.984 \pm 0.142$ & $0.786 \pm 0.077$ & $1.069 \pm 0.201$ & $0.917 \pm 0.039$ \\
\hline Chromium (Cr) & $\mu g / z$ & $2.975 \pm 2.217$ & $15.300 \pm 14.651$ & $3.835 \pm 3.144$ & $3.115 \pm 2.235$ & $2.716 \pm 1.925$ \\
\hline Ahuminim (Al) & $\mu g / g$ & $45.774 \pm 10.573$ & $40.438 \pm 12.319$ & $44.689 \pm 9.426$ & $53.332 \pm 11.701$ & $46.567 \pm 10.819$ \\
\hline Lead(P) & $\mu 8 / g$ & 4.35 & 3.815 & 3.501 & $4.428 \pm 0.887$ & 3.68 \\
\hline Manganes (Mn) & Hzjg & $0.581 \pm 0.077$ & $0.80096 \pm 0.16252$ & $0.593 \pm 0.095$ & $0.770 \pm 0.094$ & $0.574 \pm 0.078$ \\
\hline NAflel(K) & $\mu \nabla / g$ & $0.316 \pm 0.103$ & $0.1925 \pm 0.02839$ & $0.323 \pm 0.122$ & $0.300 \pm 0.073$ & $0.332 \pm 0.064$ \\
\hline Cobalt(Co) & $\mu 8 / g$ & $4.584 \pm 1.730$ & $6.313 \pm 3.443$ & $3.831 \pm 1.219$ & $6.054 \pm 3.377$ & $5.545 \pm 2.674$ \\
\hline
\end{tabular}

*Means in the same row are not significantly different ( $p>0.05)$, using ANOVA.

Table 7: Heavy metals concentration (mean $\pm \mathrm{SE})$ in gills of Clarias gariepinus collected from the investigated sites from autumn 2007 to summer 2008.

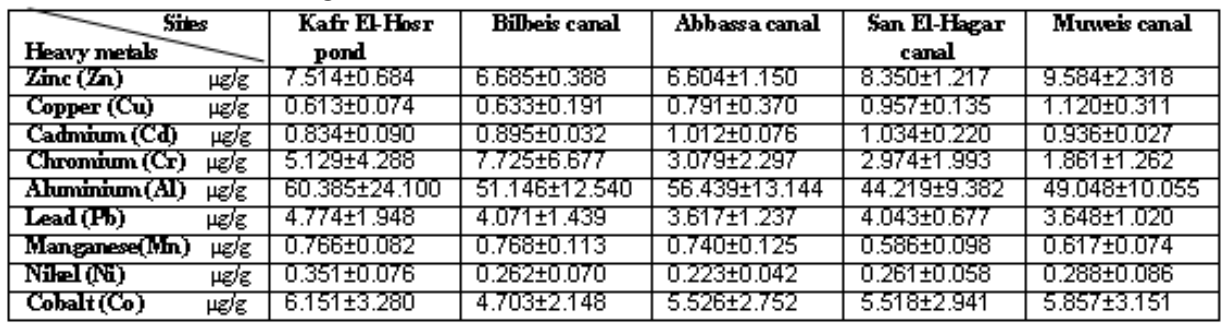

* Means in the same row are not significantly different ( $p>0.05)$, using ANOVA.

Table 8: Heavy metals concentration (mean $\pm \mathrm{SE}$ ) in gills of Bagrus bayad collected from the investigated sites from autumn 2007 to summer 2008.

\begin{tabular}{|c|c|c|c|c|c|c|}
\hline \multicolumn{2}{|c|}{ Heavy metals } & \multirow{2}{*}{$\begin{array}{l}\text { Kafr El-Hosr } \\
\text { pond } \\
5.505 \pm 2.215\end{array}$} & \multirow{2}{*}{$\begin{array}{c}\text { Bilbeis canal } \\
6.563 \pm 0.744\end{array}$} & Abbassa canal & \multirow{2}{*}{$\begin{array}{c}\text { San El-Hagar } \\
\text { canal } \\
5.113 \pm 1.612\end{array}$} & Mumeis canal \\
\hline Zleavy (Zn) & $\overline{\mu g / g}$ & & & $6.531 \pm 0.818$ & & $5.820 \pm 1.938$ \\
\hline Copper (Cu) & $\mu z / \xi$ & $0.604 \pm 0.216$ & $1.109 \pm 0.497$ & $0.550 \pm 0.124$ & $0.820 \pm 0.111$ & $0.761 \pm 0.070$ \\
\hline Cadminm (Cd) & $\mu 8 / \mathrm{g}$ & $0.824 \pm 0.002$ & $0.753 \pm 0.072$ & $0.806 \pm 0.091$ & $0.904 \pm 0.180$ & $0.979 \pm 0.209$ \\
\hline Chromium (Cr) & $\mu g / g$ & $0.556 \pm 0.264$ & $15.373 \pm 14.619$ & $2.181 \pm 1.606$ & $0.924 \pm 0.385$ & $1.736 \pm 1.051$ \\
\hline Ahuminimu(AI) & $\mu \varepsilon / \bar{g}$ & $39.455 \pm 19.945$ & 32.216 & 6.581 & $47.207 \pm 10.700$ & \pm 12.535 \\
\hline Lead(Pक) & $\mu \varepsilon / \mathrm{g}$ & $2.935 \pm 0.705$ & $3.255 \pm 0.696$ & $3.232 \pm 0.758$ & $2.421 \pm 0.205$ & $4.354 \pm 2.118$ \\
\hline Manganese ( $\mathrm{Hn}$ ) & $\mu 8 / \bar{g}$ & $0.574 \pm 0.035$ & $0.609 \pm 0.145$ & $0.587 \pm 0.109$ & $0.637 \pm 0.097$ & $0.611 \pm 0.063$ \\
\hline Nilel(it) & $\mu 8 / \delta$ & $0.223 \pm 0.198$ & $0.271 \pm 0.028$ & $0.208 \pm 0.057$ & $0.310 \pm 0.050$ & $0.267 \pm 0.069$ \\
\hline Cobalt (Co) & $\mu \nabla / g$ & $8.018 \pm 5.413$ & $4.766 \pm 2.114$ & $5.388 \pm 2.898$ & $6.881 \pm 2.779$ & $5.974 \pm 3.428$ \\
\hline
\end{tabular}

*Means with the same letters in the same row are significantly different $(\mathrm{p}<0.05)$, using ANOVA.

\section{III- Heavy metals in kidney:}

Comparing the average concentrations of heavy metals in the different study sites, (Tables 9, 10 and 11) showed variations between heavy metal concentrations in kidney. The concentrations had the order: $\mathrm{Al}>\mathrm{Zn}>\mathrm{Co}>\mathrm{Pb}>$ $\mathrm{Cr}>\mathrm{Cd}>\mathrm{Mn}>\mathrm{Cu}>\mathrm{Ni}$. 
Table 9: Heavy metals concentration (mean $\pm \mathrm{SE}$ ) in kidney of Oreochromis niloticus collected from the investigated sites from autumn 2007 to summer 2008.

\begin{tabular}{|c|c|c|c|c|c|}
\hline Heavy metals & $\begin{array}{l}\text { Kafr El-Hosr } \\
\text { pond }\end{array}$ & Bitheis canal & Abbassa canal & $\begin{array}{l}\text { San El-Hagar } \\
\text { canal }\end{array}$ & Muweis canal \\
\hline Znc ( $(\mathrm{Zn})$ & $15.150 \pm 9.023$ & $6.784 \pm 0.962$ & $6.621 \pm 0.855$ & $6.638 \pm 0.851$ & $7.354 \pm 0.557$ \\
\hline Copper (Cu) & $0.750 \pm 0.086$ & $1.118 \pm$ & 0.92 & $2.630 \pm 1.437$ & 1.98 \\
\hline Cadmium (Cd) $\mu / g$ & $0.938 \pm 0.080$ & 1.024 & 0.86 & $1.112 \pm 0.207$ & .151 \\
\hline Chromin (Cr) & $3.811 \pm 2.950$ & $15.624 \pm 14898$ & 4.024 & $3.336 \pm 2.256$ & 2.86 \\
\hline Ahomín் (AI) & $48.516 \pm 11.626$ & 43.249 & 1.290 & $57.421 \pm 13.007$ & 12.367 \\
\hline Lead(Pक) & $4.469 \pm 1.957$ & 4.261 & 3.80 & $4.505 \pm 0.888$ & 0.682 \\
\hline Manganese (Mn) $\mu g / g$ & 0.65 & 0.85 & 0.6. & $0.846 \pm 0.111$ & 097 \\
\hline NHm (r) & $0.364 \pm 0.103$ & 0.22 & 0.37 & $0.338 \pm 0.071$ & \pm 0.072 \\
\hline Cobalt(Co) & $5.665 \pm 2.692$ & 3.605 & $5.631 \pm 2.809$ & 6.35 & $5.767 \pm 2.673$ \\
\hline
\end{tabular}

*Means in the same row are not significantly different ( $p>0.05)$, using ANOVA.

Table (10): Heavy metals concentration (mean $\pm \mathrm{SE}$ ) in kidney of Clarias gariepinus collected from the investigated sites from autumn 2007 to summer 2008.

\begin{tabular}{|c|c|c|c|c|c|}
\hline Heavy metals & $\begin{array}{l}\text { Kafr El-Hosr } \\
\text { pond }\end{array}$ & Bilbeis canal & Abbassa canal & $\begin{array}{c}\text { San El-Hagar } \\
\text { canal }\end{array}$ & Mureis canal \\
\hline Zhinc ( $(\mathrm{G}) \quad \mu g / g$ & $9.378 \pm 0.985$ & $7.662 \pm 0.387$ & $8.473 \pm 1.247$ & $8.822 \pm 1.240$ & $10.194 \pm 2.040$ \\
\hline Copper (Cu) & $0.920 \pm 0.134$ & $1.335 \pm 0.216$ & $1.327 \pm 0.490$ & $2.818 \pm 1.498$ & $1.981 \pm 0.859$ \\
\hline Cadminu(Cd) $\mu g / g$ & $0.864 \pm 0.096$ & $1.030 \pm 0.073$ & $1.060 \pm 0.084$ & $1.077 \pm 0.229$ & $1.031 \pm 0.067$ \\
\hline Chromium(Cr) $\mu g / g$ & $5.517 \pm 4.555$ & $10.246 \pm 9.026$ & $3.694 \pm 2.851$ & $3.440 \pm 2.309$ & $2.378 \pm 1.610$ \\
\hline Ahminim(A) $\mu g / g$ & $64.478 \pm 25.783$ & $55.568 \pm 13.530$ & $59.661 \pm 14.106$ & $46.702 \pm 9.778$ & $51.468 \pm 10.483$ \\
\hline Lead(P5) & $5.014 \pm 2.005$ & $4.398 \pm 1.399$ & $4.945 \pm 2.121$ & $4.554 \pm 1.002$ & $4.148 \pm 1.251$ \\
\hline Manganes (Mn) $\mu g / g$ & $0.804 \pm 0.074$ & $0.865 \pm 0.156$ & $0.847 \pm 0.125$ & $0.647 \pm 0.113$ & $0.658 \pm 0.087$ \\
\hline NAm(t) & $0.398 \pm 0.086$ & $0.302 \pm 0.066$ & $0.301 \pm 0.062$ & $0.354 \pm 0.058$ & $0.328 \pm 0.066$ \\
\hline Cobalt (Co) & $6.296 \pm 3.316$ & $4.949 \pm 2.098$ & $5.757 \pm 2.896$ & $6.353 \pm 3.488$ & $6.275 \pm 3.425$ \\
\hline
\end{tabular}

*Means in the same row are not significantly different ( $p>0.05)$, using ANOVA.

Table (11): Heavy metals concentration (mean \pm SE) in kidney of Bagrus bayad collected from the investigated sites from autumn 2007 to summer 2008.

\begin{tabular}{|c|c|c|c|c|c|c|}
\hline Heavy metals & & $\begin{array}{l}\text { Kafr El-Hosr } \\
\text { pond }\end{array}$ & Billbeis canal & Abbassa canal & $\begin{array}{l}\text { San El-Hagar } \\
\text { canal }\end{array}$ & Murueis canal \\
\hline Zinc ( $(\mathbf{G})$ & $\mu g / g$ & $6.323 \pm 1.848$ & $7.456 \pm 0.326$ & $7.303 \pm 0.834$ & $5.496 \pm 1.723$ & $6.187 \pm 2.056$ \\
\hline Copper (Cu) & $\mu b / \mathrm{g}$ & $0.859 \pm 0.406$ & $2.416 \pm 1.410$ & $1.503 \pm 0.770$ & $1.196 \pm 0.267$ & $0.878 \pm 0.103$ \\
\hline Cadmium (Cd) & $\mu g / g$ & $0.883 \pm 0.050$ & $0.838 \pm 0.021$ & $0.955 \pm 0.069$ & $1.068 \pm 0.146$ & $1.064 \pm 0.204$ \\
\hline Chromium (CF) & $\mu \nabla / g$ & $0.607 \pm 0.268$ & $15.647 \pm 14.784$ & $2.658 \pm 1.937$ & $1.193 \pm 0.360$ & $2.956 \pm 2.199$ \\
\hline Ahumíníum (AI) & 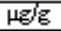 & $43.830 \pm 23.04$ & $38.962 \pm 10.018$ & $54.092 \pm 16.662$ & $51.731 \pm 11.314$ & $67.919 \pm 19.193$ \\
\hline Lead(Pक) & $\mu g / \mathrm{g}$ & $3.105 \pm 0.59$ & $4.134 \pm 1.216$ & $3.492 \pm 0.712$ & $2.978 \pm 0.230$ & $4.455 \pm 2.077$ \\
\hline Manganese (hin) & $\mu g / g$ & $0.602 \pm 0.029$ & $0.686 \pm 0.151$ & $0.661 \pm 0.140$ & $0.674 \pm 0.094$ & $0.650 \pm 0.062$ \\
\hline Nife (14) & $\mu \delta / \xi$ & $0.237 \pm 0.200$ & $0.295 \pm 0.033$ & $0.244 \pm 0.063$ & $0.374 \pm 0.042$ & $0.296 \pm 0.081$ \\
\hline Cobalt(Co) & $\mu g / g$ & $8.063 \pm 5.533$ & $4.968 \pm 2.248$ & $6.116 \pm 3.369$ & $7.537 \pm 2.788$ & $6.175 \pm 3.419$ \\
\hline
\end{tabular}

*Means in the same row are not significantly different ( $p>0.05)$, using ANOVA.

\section{IV- Heavy metals in liver tissue:}

Comparing the average concentrations of heavy metals in the different study sites, (Tables 12, 13 and 14) and Fig. (3, 4, 5, 6 and 7) showed variations between heavy metal concentrations in liver tissue. The concentration was as seen in the previous studied tissues $\mathrm{Al}>\mathrm{Zn}>\mathrm{Co}>\mathrm{Pb}>\mathrm{Cr}>\mathrm{Cd}>\mathrm{Mn}>\mathrm{Cu}>$ $\mathrm{Ni}$. 
Table 12: Heavy metals concentration (mean $\pm \mathrm{SE}$ ) in liver tissues of Oreochromis niloticus collected from the investigated sites from autumn 2007 to summer 2008.

\begin{tabular}{|c|c|c|c|c|c|}
\hline Heavy metals & $\begin{array}{l}\text { Kafr El-Hosr } \\
\text { pond }\end{array}$ & Billeis canal & Abbassa canal & $\begin{array}{l}\text { San El-Hagar } \\
\text { canal }\end{array}$ & Mumeis ca \\
\hline Zinc (Zn) & $17.941 \pm 9.869$ & $9.114 \pm 1.451$ & $8.685 \pm 0.636$ & $7.944 \pm 0.689$ & $8.260 \pm 0.697$ \\
\hline Copper (Cu) & $3.995 \pm 0.809$ & $12.484 \pm 4.688$ & $17.256 \pm 8.059$ & $15.720 \pm 5.941$ & $12.979 \pm 8.154$ \\
\hline Cadminm (Cd) & $1.015 \pm 0.062$ & $1.123 \pm 0.229$ & $1.00 \%$ & $1.168 \pm 0.210$ & $1.269 \pm 0.220$ \\
\hline Chrominm (C) $\mu g / g$ & $6.545 \pm 5.342$ & 16.6 & 4.15 & $4 \pm 3.535$ & \\
\hline Ahomíním (AI) $\mu g / g$ & $52.948 \pm 13.003$ & 48.9 & .776 & $95 \pm 17.927$ & 4.050 \\
\hline Lead(Pक) & $5.699 \pm 3.048$ & 4.71 & 4.25 & $4.686 \pm 0.954$ & 475 \\
\hline Manganese(Mn) $\mu g / g$ & $0.832 \pm 0.131 a$ & 0.95 & 0.8 & $1.375 \pm 0.267 a b$ & 0.9 \\
\hline NHF (16) & $0.440 \pm 0.115$ & & & \pm 0.069 & 0.4 \\
\hline Cobalt(Co) & $6.041 \pm 2.827$ & 6.88 & 5.9 & $6.870 \pm 3.791$ & 6.5 \\
\hline
\end{tabular}

*Means with the same letters in the same row are significantly different $(\mathrm{p}<0.05)$, using ANOVA.

Table 13: Heavy metals concentration (mean $\pm \mathrm{SE}$ ) in liver tissues of Clarias gariepinus collected from the investigated sites from autumn 2007 to summer 2008.

\begin{tabular}{|c|c|c|c|c|c|}
\hline Heavy metals & $\begin{array}{l}\text { Kafr El-Hosr } \\
\text { pond }\end{array}$ & Billeis canal & Abbassa canal & $\begin{array}{l}\text { San El-Hagar } \\
\text { canal }\end{array}$ & Murueis canal \\
\hline Znc (Zn) $\quad \mu g / g$ & $12.575 \pm 1.485$ & $11.247 \pm 0.973$ & $12.996 \pm 3.053$ & $15.404 \pm 1.469$ & $14.536 \pm 2.778$ \\
\hline Copper (Cu) & $4.117 \pm 0.802$ & $3.846 \pm 1.090$ & $8.009 \pm 3.676$ & $9.963 \pm 2.114$ & $9.579 \pm 3.567$ \\
\hline Cadminm (Cd) & $0.932 \pm 0.092$ & $1.097 \pm 0.111$ & $1.236 \pm 0.193$ & $1.206 \pm 0.232$ & $1.216 \pm 0.167$ \\
\hline Chromíum (C) $\mu \mathrm{g} / \mathrm{g}$ & $7.666 \pm 5.930$ & $15922 \pm 14385$ & $4.474 \pm 3.300$ & $6.853 \pm 5.351$ & $3.748 \pm 2.883$ \\
\hline Ahuminium (AI) $\mu g / g$ & $67.871 \pm 25.802$ & $64.154 \pm 16.237$ & $67.696 \pm 15.574$ & $66.971 \pm 17.047$ & $59.977 \pm 13.610$ \\
\hline Lead(P) & $5.996 \pm 2.827$ & $5.124 \pm 1.563$ & $5.656 \pm 2.274$ & $4.891 \pm 1.095$ & $5.057 \pm 1.638$ \\
\hline Manganese (Mn) $\mu g / g$ & $0.992 \pm 0.063$ & $1.028 \pm 0.184$ & $0.963 \pm 0.160$ & $0.887 \pm 0.162$ & $0.738 \pm 0.079$ \\
\hline NAfel(N) & $0.508 \pm 0.108$ & $0.381 \pm 0.048$ & $0.438 \pm 0.069$ & $0.428 \pm 0.077$ & $0.407 \pm 0.050$ \\
\hline Cobalt(Co) & $6.651 \pm 3.411$ & $5.655 \pm 2.270$ & $6.263 \pm 3.232$ & $6.986 \pm$ & $6.649 \pm 3.624$ \\
\hline
\end{tabular}

*Means in the same row are not significantly different ( $\mathrm{p}>0.05)$, using ANOVA.

Table 14: Heavy metals concentration (mean $\pm \mathrm{SE}$ ) in liver tissues of Bagrus bayad collected from the investigated sites from autumn 2007 to summer 2008.

\begin{tabular}{|c|c|c|c|c|c|c|}
\hline Heavy metals & & $\begin{array}{l}\text { Kafi El-Hos } r \\
\text { poind }\end{array}$ & Bilbeis canal & Abbassa canal & $\begin{array}{l}\text { San El-Hagar } \\
\text { canal }\end{array}$ & Hureis canal \\
\hline$Z_{\text {me }}[\mathrm{Zn}]$ & 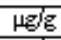 & $7.696 \pm 0.985$ & $15.766 \pm 5.112 a$ & $12.074 \pm 1.281$ & $6.300 \pm 2.018 \mathrm{a}$ & $8.697 \pm 3008$ \\
\hline Copper [Cu] & $\mu b 5$ & $1.224 \pm 0.356$ & $3.963 \pm 1.568$ & $3.086 \pm 0.622$ & $5.191 \pm 1.506$ & $3.013 \pm 0.943$ \\
\hline Cadminm (Cd) & 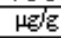 & $0.934 \pm 0.036$ & $0.965 \pm 0.0108$ & $7.1940 \pm 0.160$ & $1.164 \pm 0.171$ & $1.214 \pm 0.226$ \\
\hline Chrominm (Cr) & $\mu g / g$ & $1.114 \pm 0.681$ & $16.626 \pm 15.320$ & $3.342 \pm 2.496$ & $1.411 \pm 0.373$ & $4.026 \pm 3.168$ \\
\hline Ahominimu (A1] & $\mu \boxminus \bar{\varepsilon}$ & $48.012 \pm 25.798$ & $55.336 \pm 7$ न 988 & $59.542 \pm 78.685$ & $59.553 \pm 73978$ & $86.284 \pm 30.520$ \\
\hline Lead $(\mathbf{P})]$ & $\mu g / 5$ & $3.113 \pm 0.553$ & $4.320 \pm 1.165$ & $4.237 \pm 0.968$ & $3.276 \pm 0.232$ & $5.025 \pm 2.168$ \\
\hline hanganese[(hin] & $\mu E / \bar{g}$ & $0.733 \pm 0.015$ & $0.783 \pm 0.147$ & $0.807 \pm 0.146$ & $0.903 \pm 0.742$ & $0.810 \pm 0.124$ \\
\hline Nisel[1] & $18 j$ & $0.37 \pm 0.103$ & 0.38000.0398 & $0.294 \pm 0.065$ & $0.472 \pm 0.083$ & $0.337 \pm 0.092$ \\
\hline Cohalt [Co] & $\mu \xi \xi$ & $8.483 \pm 5.898$ & $5.298 \pm 2.476$ & $6.478 \pm 3.484$ & $8.471 \pm 3.749$ & $6.564 \pm 3.602$ \\
\hline
\end{tabular}

*Means with the same letters in the same row are significantly different $(p<0.05)$, using ANOVA. 


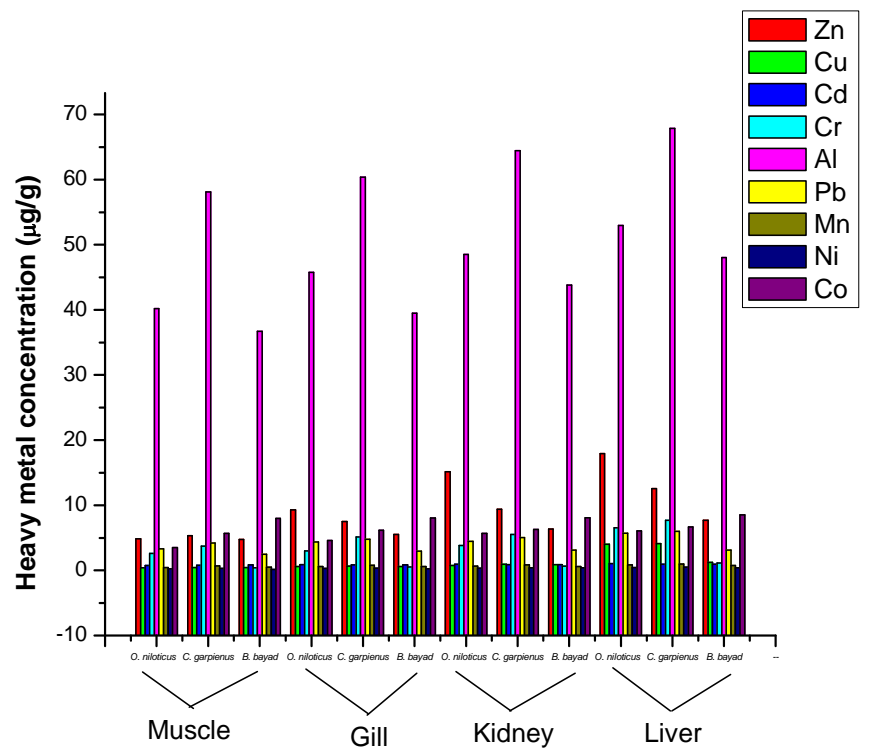

Fig. 3: Heavy metals concentrations (annual mean \pm SE) in muscle, gills, kidney and liver tissues of Oreochromis niloticus, Clarias gariepinus and Bagrus bayad at Kafr El-Hosr pond during autumn 2007-summer 2008.

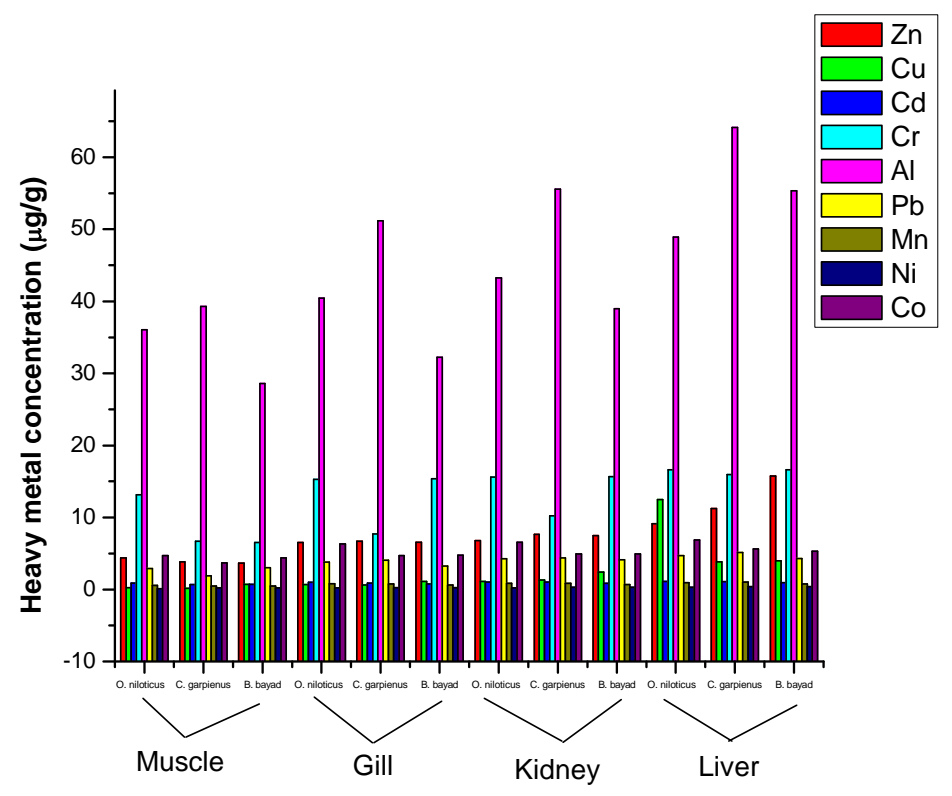

Fig. 4: Heavy metals concentrations (annual mean \pm SE) in muscle, gills, kidney and liver tissues of Oreochromis niloticus, Clarias gariepinus and Bagrus bayad at Bilbeis canal during autumn 2007-summer 2008. 


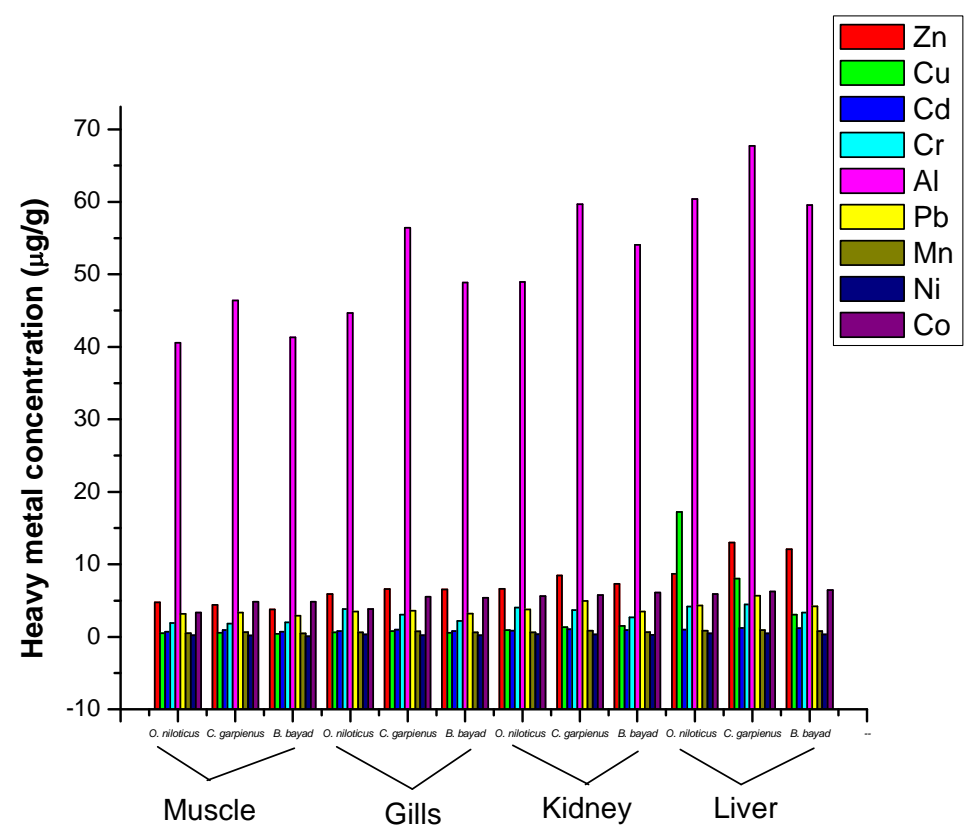

Fig. (5): Heavy metals concentrations (annual mean $\pm \mathrm{SE}$ ) in muscle, gills, kidney and liver tissues of Oreochromis niloticus, Clarias gariepinus and Bagrus bayad at Abbassa canal during autumn 2007-summer 2008.

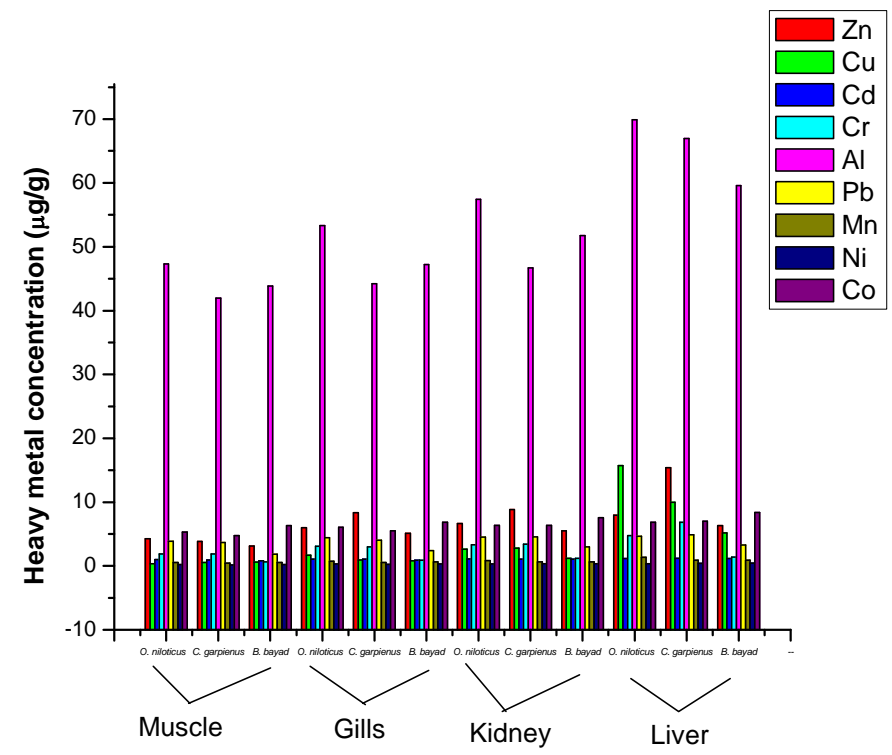

Fig. 6: Heavy metals concentrations (annual mean \pm SE) in muscle, gills, kidney and liver tissues of Oreochromis niloticus, Clarias gariepinus and Bagrus bayad at San El-Hagar canal during autumn 2007-summer 2008. 


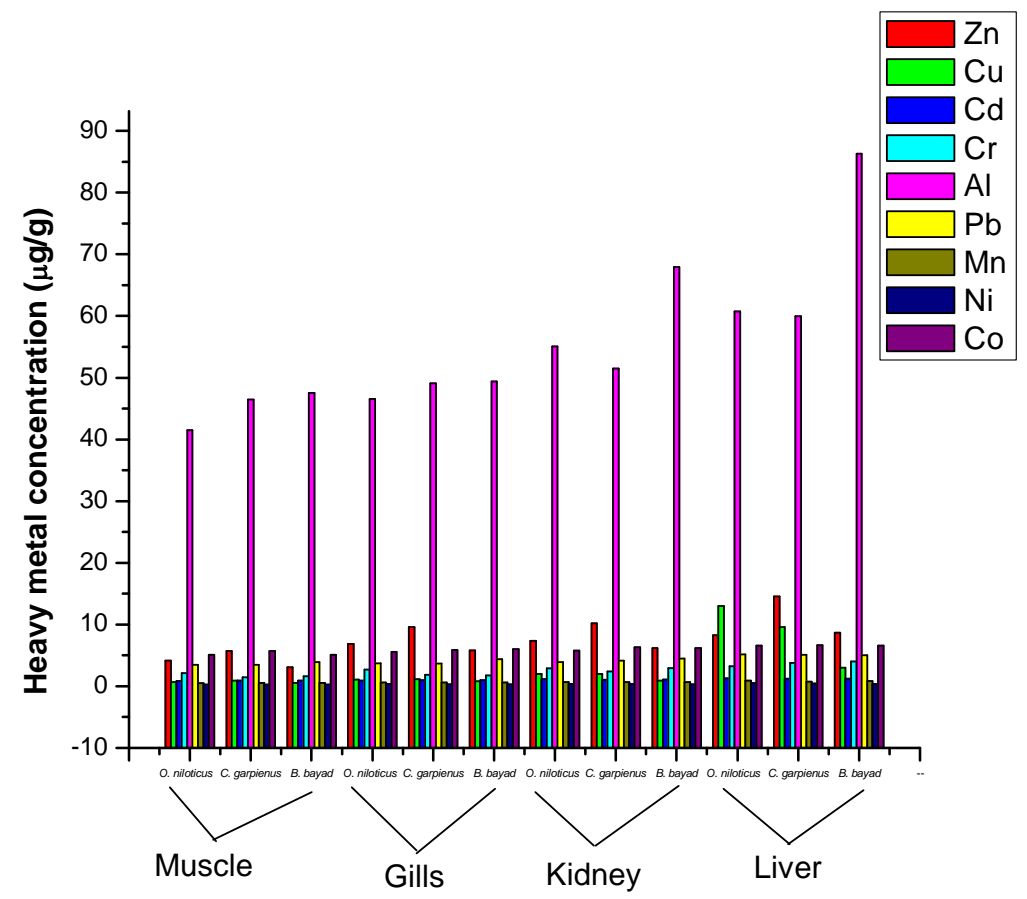

Fig. 7: Heavy metals concentrations (annual mean \pm SE) in muscle, gills, kidney and liver tissues of Oreochromis niloticus, Clarias gariepinus and Bagrus bayad at Muweis canal during autumn 2007-summer 2008.

\section{Heavy metals in water:}

\section{DISCUSSION}

For all heavy metals, the maximum concentrations were observed in spring and winter, while the minimum values were detected in summer and autumn. These results comply with those recorded by Saeed (2000) who found that heavy metals concentration showed seasonal variations, being greater in winter and lowest in summer. This may be attributed to the phytoplankton growth which was higher in summer and autumn seasons that can absorb large quantities of heavy metals from water. Moreover, the highest concentrations of all heavy metals were detected in water samples collected from Muweis canal [especially for zinc where zinc oxide is used as oxidizing agent to produce fatty acids) and chromium (chromium oxide is used as inorganic color additive) which are used in soap manufacture], Kafr El-Hosr pond and San El-Hagar canalwhich may be attributed to wastes of industrial activities and wastewater.

\section{Heavy metals in sediment:}

Concerning the mean concentrations of heavy metals $\mathrm{Zn}, \mathrm{Cu}, \mathrm{Pb}, \mathrm{Cd}, \mathrm{Cr}$, $\mathrm{Mn}, \mathrm{Ni}, \mathrm{Co}$ and $\mathrm{Al}(<120,<16,<31,<0.6,<26,<460,<16,19$ and $100 \mu \mathrm{g} / \mathrm{g}$ respectively), the levels were below the permissible limits reported by Ontario Ministry of the Environment (ANZECC, 2000), Shall standard [after Foerstner 
\& Wittmann (1979)] for cobalt, and Hamilton and Buhl (2003) for aluminium, at all study sites, and were accepted by Saeed and Shaker (2010) and Olubunmi (2010).

\section{Heavy metals in fish tissues: \\ Muscle tissue:}

The present study revealed that $\mathrm{Pb}, \mathrm{Cd}, \mathrm{Cr}, \mathrm{Al}$ and $\mathrm{Co}$ were higher than the permissible levels recommended by WHO (1984 \& 1993), USEPA (1986), ANZECC (2000) and Abbasi et al. (1997) [2.0, 0.5, 0.15, 0.1 and $1.2 \mu \mathrm{g} / \mathrm{g}$ respectively]. Although the results were higher than those recorded by Dobaradaran et al. (2010), the data were nearly similar to those of Schlotfeldt and Alderman (1995), Uluozlu et al. (2007), Mohamed (2008), Miclean et al. (2009) and Saeed and Shaker (2010). On the other hand, Zn, Cu, Mn and Ni levels were lower than permitted limits $(50,20,2.5$ and $0.4 \mu \mathrm{g} / \mathrm{g}$ respectively), but still lesser than those reported by Gabriel et al. (2006), Morshdy et al. (2007), Al-Bader (2008), Dobaradaran et al. (2010) and Saeed and Shaker (2010). These variations may be attributed to the differences between the localities, and the amount and source of pollution from an area to another.

\section{Gills, Kidney and liver tissues:}

From the present data, it is obvious that liver, kidney and gills accumulated higher concentrations of the studied heavy metals than the muscles. The mean of all studied metals of these tissues collected from the study sites exceeded the permissible concentrations (except for $\mathrm{Zn}, \mathrm{Cu}, \mathrm{Mn}$ and $\mathrm{Ni}$ ). Liver represented the highest site of concentrations of heavy metals followed by kidney then gills and finally muscle tissue. This may be attributed to the major role of liver in detoxification and protection from heavy metal exposure, both by producing metallothionines (metal binding-proteins) and by acting as storage site for bound metals (Pratap et al., 1989). Also, the liver concentrates these metals from the blood circulatory system of the fish. The present results are in agreement with Velcheva (2006) who reported that heavy metals were significantly higher in fish viscera, including liver tissue and kidney than in the edible muscle tissue. The high heavy metal content in gills of fish collected from the three sources of water can be related to accumulation of such heavy metals from the water primarily through fish gill where metallothionine enhances that bioaccumulation in gills and its uptake could be controlled by the amount of water passing through the gills (Saeed, 2000).

Fish collected from area of industrial activity (Muweis canal) and swage areas (Kafr El-Hosr pond and San El-Hagar canal) had significantly greater concentrations of heavy metals than fish collected from areas of agricultural activities (Bilbeis canal and Abbassa canal). These variations in heavy metal concentrations may be due to the nature of water source. Moreover, it is obvious that the average heavy metal concentrations in different tissues of Clarias gariepinus were higher than those of Oreochromis niloticus and Bagrus bayad. This may be due to the difference of feeding habits of the three species, where 
the former fish is mainly omnivorous feeding on fish, insect larvae, mollusks, planktonic organisms and water weeds (Bishai and Khalil, 1997) which accumulate large amounts of heavy metals (Rizkalla and Abou-Donia, 1996).

Also, Oreochromis niloticus feeds mainly on phytoplankton (Schroeder, 1983) which accumulate large amounts of heavy metals while Bagrus bayad feeds mainly on fishes, insects and crustaceans. Moreover, Clarias gariepinus lives mainly in muddy or semi-muddy bottom (Bishai and Khalil, 1997) and Oreochromis niloticus wanders in water from surface to bottom, being frequently in contact with soil particles (Saeed, 2000), while Bagrus bayad lives in deep water in crevices of rocks at the daylight (Bishai and Khalil, 1997).

\section{CONCLUSION AND RECOMMENDATION}

From the present results, it can be concluded that Conditions at industrial drainage, sewage wastewater and agriculture drainage fish are not safe for human consumption since the heavy metal analysis revealed a public health hazard as the quality of fish grown in such water did not comply with the standards levels recommended by WHO, USEPA and ANZECC. Potential adverse health effects in such application could be avoided if the wastewater is sufficiently treated.

\section{REFERENCES}

Abbasi, S. A.; Abbasi, N. and Soni, R. (1997). Heavy metals in the environment. Showroom, pp. 1-297.

Abdel-Hafez, S. M. and El-Caryony, I. A. (2009). An economic study on the production of catfish in the Egyptian fisheries. J. Arab. Aqua. Soc., 4 (1): 19-34.

Akubugwo, I. E.; Ofoegbu, C. J. and Ukwuoma, C. U. (2007). Physicochemical studies on Uburu Salt Lake Ebonyi Staste-Nigeria. Pak. J. Biol. Sci., 10(17): 3170-3174.

Al- Bader, N. (2008). Heavy metal levels in most common available fish species in Saudi market. II J. Food Tech., 6(4): 173-177.

Allinson, G.; Laurenson, L. J. B.; Pistone, G.; Stagnitti, F. and Jones, P. L. (2000). Effects of dietary copper on the Australian freshwater crayfish, Cherax destructor, 46: 117-123.

American Public Health Association (APHA) (1985). Standard methods for the examination of water and wastewater. $16^{\text {th }}$ ed., Washington, D. C. 
ANZECC (2000). Australian and New Zealand guidelines for fresh and marine water quality. Australian and New Zealand Environmental Conservation Council \& Agriculture and Resource Management Council of Australian and New Zealand. Canberra.pp. 1-123.

Begum, A.; Harikrishna, S. and Khan, I. (2009). Analysis of heavy metals in water, sediment and fish samples of Madivala Lake of Bangalore, Karnataka. Int. J. of Chem Tech Res., 1(2):245-249.

Bishai, H. M. and Khalil, M. T. (1997). Freshwater fishes of Egypt. Publication of National Biodiversity Unit, 9:1-229.

Bishop, O. V. (1980). A practical guide for the experimental biologist. In "Statistics for Biology", Bishop, O. V. (ed.), $3^{\text {rd }}$ edition, Longman group limited., 28 pp.

Boyd, C. E. (1990). Water quality in ponds for aquaculture. Alabama Agriculture Experiment Station, Auburn Univ., Alabama, U. S. A.

Burger, J.; Gaines, K. F.; Boring, S.; Stephens, L.; Snodgrass, J.; Dixon, C.; McMahon, M.; Shukla, S.; Shukla, T. and Gochfeld, M. (2002). Metal levels in fish from the Savannah River: Potential hazards to fish and other receptors. Environ. Res., 89: 85-97.

Dallinger, R.; Prosi, F.; Segner, H. and Black, H. (1987). Contaminated food and uptake of heavy metals by rainbow trout (Salmo gairdneri): A field study. Oecologia. 73: 91-98.

Dobaradaran, S.; Naddafi, K.; Nazmara, S. and Ghaedi, H. (2010). Heavy metals $(\mathrm{Cd}, \mathrm{Cu}, \mathrm{Ni}$ and $\mathrm{Pb})$ content in two fish species of Persian Gulf in Bushehr Port, Iran. Af. J. of Biotech., 9(37):6191- 6193.

Fernandez,-Leborans, G. and Olalla-Herrero, Y. (2000). Toxicity and bioaccumulation of lead and cadmium in marine protozoa communities. Ecotoxicol. and Environ. Saf.. 47: 266-276.

Forstner, U. and Wittman, G. T. W. (1979). Metal pollution in aquatic environment. Springer-Verlag Berlin, Heidelerg, PP. 486.

Gabriel, O.; Rita, O.; Clifford, A.; Cynthia, O.; Harrison, N. and Kennedy, K. (2006). Metal pollution of fish of Qua-Iboe River Estuary: Possible implications for neurotoxicity. The Int. J. of Toxicol., 3(1): 1-6. 
Gupta, P. K. (2000). Methods in environmental analysis water, soil and air.Agrobios, 5: 1- 400.

Hamilton, S. J. and Buhl, K. J. (2003). Selenium and other trace elements in water, sediment, Aquatic plants, Aquatic invertebrates, and fish from streams in southeastern Idaho near phosphate mining operations: September 2000. USGS science for a changing world, pp. 1-67.

Langston, W. J.; Burt, G. R. And Pope, N. D. (1999). Bioavailability of metals in sediments of the dogger bank (Central North Sea): A mesocosm study. Estuarine, Coastal and Shelf Science. 48: 519-540.

Linnik, P. M. and Zubenko, I. B. (2000). Role of bottom srdiments in the secondary pollution of aquatic environments by Heavy-metal compounds. Lakes and Reservoirs: Res. and Man., 5: 11-21.

McCreadie, J. W.; Alder, P. H.; Grillet, M. E. and Hamada, N. (2006). Sampling and statistics in understanding distributions of black fly larvae (Diptera: Simuliidae). Acta Entomological Serbica, 11: 89-96.

Miclean, M. I.; Ştefãnescu, L. N.; Levei, E. A.; Şenilã, M.; Mãrginean, S. F.; Romam, C. M. and Cordoş, E. A. (2009). Ingestion induced health risk in surface waters near tailings ponds (North-Western Romania). AACL Bioflux, 2(3): 275-283.

Mohamed, F. A. S. (2008). Bioaccumulation of selected metals and histopathological alterations in tissues of Oreochromis niloticus and Lates niloticus from Lake Nasser, Egypt. Glob. Vet., 2(4): 205-218.

Mohan, B. S. and Hosetti, B. B. (1999). Aquatic plants for toxicity assessment. Environ. Res., 81: 259-274.

Morshdy, A.; El-Dosky, K. E. and El-shebaie, S. (2007). Some heavy metal residues in mackerel and saurus fish. Zag. Vet. J., 35(4): 114-120.

Olaifa, F. E.; Olaifa, A. K.; Adelaja, A. A. and Owolabi, A. G. (2004). Heavy metal contamination of Clarias gariepinus from a lake and fish from farm in Ibadan, Nigeria. A f. J. of Biomed. Res., 7: 145-148.

Olubunmi, F. E. (2010). Evaluation of the status of heavy metal pollution of sediment of Agbabu Bitumen Deposit Area, Nigeria. Eur. J. of Sci. Res., 41(3):373-382. 
Pratap, H. B.; Lock, R. A. C. and Bonga, S. E. W. (1989). Effect of waterborne and dietary cadmium on plasma ions of the teleost Oreochromis mossambicus in relation to water calcium levels. Arch. Environ. Contam. Toxicol., 18(4): 568-575.

Rakocy, J. E. (2011). Oreochromis niloticus. FAO. In Cultured aquatic species fact sheets. Edited and compiled by Valerio Crespi and Michael New. CD-ROM (multilingual).

Ramelow, G. J.; Webre, C. L.; Mueller, C. L.; Beck, J. N.; Young, J. C. and Langley, M. P. (1989). Variations of heavy metals and arsenic in fish and other organisms from the Calcasien River and Lake, Louisiana. Archive of Environ. Contam. and Toxicol., 18: 804-818.

Raymus-Keller, A.; Olson, K. E.; McGaw, M.; Oray, C.; Carlson, J. O. and Beaty, B. J. (1998). Effect of heavy metals on Aedes aegypti (Diptera: Culicidea) larvae. Ecotoxicol. and Environ.saf., 39: 41-47.

Rizkalla, E. and Abo-Donia, M. A. (1996). Some industrial heavy metal pollutants in River Nile and their effect on biological and non-biological inhabitants. 1. Essential heavy metals. Egypt, J. Agric. Res., 74 (1): 225237.

Saeed, S. M. (2000). A study on factors affecting fish production from certain fish farms in the Delta. M. Sc.Thesis. Faculty of Science, Ain Shams University, Egypt.

Saeed, S. M. and Shaker, I. M. (2010). Assessment of heavy metals pollution in water and sediments and their effect on Oreochromis niloticus in the northern Delta Lakes, Egypt. $8^{\text {th }}$ Intern. Symp. on Tilapia in Aqua., pp. 475- 489 .

Schlotfeldt, H. J. and Alderman, D. J. (1995). What should I do? A practical guide for the freshwater fish farmer. European Association of Fish Pathologists/Warwick Press, Weymouth.

Schroeder, G. L. (1983). Sources of fish and prawn- growth in polyculture ponds as indicated by delta $\mathrm{C}$ analysis Aqua., 35: 29-42.

Uluozlu, O. D.; Tuzen, M.; Mendil, D. and Soylak, M. (2007). Trace metal content in nine species of fish from the Black and Aegean Seas, Tur. Food Chem., 104 (2): 835-840. 
United States Environmental Protection Agency (USEPA) (1986). Quality Criteria for Water. EPA/ 440/5-86-001.

Van den Broek, J. L.; Gledhill, K. S. and Morgan, D. G. (2002). Heavy metal concentration in the mosquito fish, Gambusia holbrooki, in the Manly Lagoon Catchment. In: UTS freshwater ecology report 2002, Department of Environmental Science, University of Technology, Sydney.

Velcheva, I. G. (2006). Zinc content in the organs and tissues of freshwater fish from the Kardjali and Studen Kladenets Dam Lakes in Bulgaria. Turk. J. Zool., 30: 1-7.

Wildianarko, B.; Van Gestel, C. A. M.; Verweij, R. A. and Van Straalen, N. M. (2000). Associations between trace metals in sediment, water and guppy, Poecilia reticulate (Peters), from Urban streams of Semarang, Indonesia. Ecotoxicol. and Environ. Saf.. 46: 101-107.

Wong, P. T. S. and Dixon, D. G. (1995). Bioassessment of water quality. Environ. Toxicol.and Water Qual., 10: 9-17.

World Health Organization (WHO) (1984). Guide lines for drinking water quality. Geneva.

World Health Organization (WHO) (1993). Revision of WHO guidelines for water quality.WHO. Geneva. 